\title{
INTERACTIVE MEDIA OF DYNAMIC SKETCH
}

\author{
Kuan-Ting Chen \\ Graduate School of Computational \\ Design \\ National Yunlin University of Science \\ \& Technology \\ 123 University Road, Section 3, \\ Douliou, Yunlin 64002, Taiwan \\ austin@mail.gcd.yuntech.edu.tw
}

\author{
Teng-Wen Chang \\ Graduate School of Computational \\ Design \\ National Yunlin University of Science \\ \& Technology \\ 123 University Road, Section 3, \\ Douliou, Yunlin 64002, Taiwan \\ tengwen@yuntech.edu.tw
}

\author{
Shang-Chia Chiou \\ Graduate School of Computational \\ Design \\ National Yunlin University of Science \\ \& Technology \\ 123 University Road, Section 3, \\ Douliou, Yunlin 64002, Taiwan \\ chiousc@yuntech.edu.tw
}

Abstract: Our goal is to provide a Multi-Perspective viewpoint interactive interface media of sketch-based for rapid get spatial layout in Show-Window. In traditional way, designer is used to describe concept in early phases of design by Single-Perspective viewpoint via sketch in Show-Window design environment. It usually makes blind spot of spatial vision in early phases of design because it can't break the Single-Perspective viewpoint. Therefore we present an interactive media of sketch-based that can get Multi-Perspective viewpoint in early phases of design to support designer. Show-Window designer not only can have synchronic interaction with object of the design environment, and have more design thinking and variability of spatial layout but also can immediate edit, revise the vision and generate an optimum spatial layout again, and reduce time cost of later phases of design by design sketch after via the interactive media.

Keywords: Multi-Perspective, Sketch, Show-Window, Interactive media

\section{INRODUCTION}

\subsection{Deign Process}

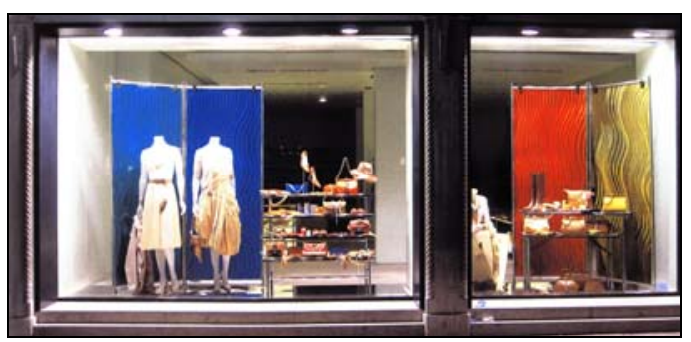

Figure 1. The Show-Window[1]

In Show-Window ${ }^{1}$ [2] (Figure 1) design environment, through interviews we know that the part costs designer the most of time and needs to be discussed is the early phases of design conceptual development. In the category of Show-Window design, conceptual design plays a very important role, because the significance of Show-Window display is to show idea that we do not know or to reinterpret something we already know[3]. This phase is not only the most important step in the early design but a vital key-point to affect the later phase of design process. However, in the early phases of design conceptual process, we usually discuss and record design concept via media of sketch.

\subsection{Consumers’ Behavior}

According to the statistics[4], 24\% customers are attracted by Show-Window to enter stores. So how to use

\footnotetext{
${ }^{1}$ Show-Window is a medium space between insides and outside of store
}

Show-Window to express creativity, attract customers, expose products, and impress customers the stores are usually the focus that Show-Window designer want to highlight. One way to have customers' attention is to use human viewpoint and then to get their watchful eyes[5,6]. In Show-Window environment, customers usually look around the Show-Window in a dynamic state. They usually examine Show-Window in different perspective views in different viewpoint (Figure 2).

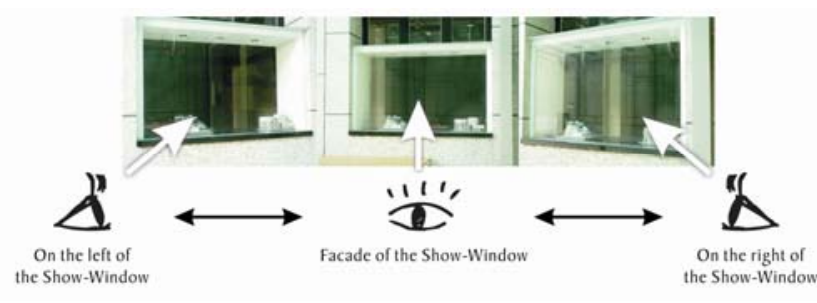

Figure 2. Three perspective views of consumers' while they are moving

Nevertheless, the interaction between the customers and Show-Window cannot be imagined, simulated, and used as a reference when Show-Window designer design their conceptual design sketch.

\subsection{Single-Perspective}

In traditional way, designer is used to describe concept in early phases of design by Single-Perspective viewpoint via sketch in Show-Window design environment. It usually makes blind spot of spatial vision in early phases of design because it can't break the Single-Perspective viewpoint. In the past, we usually used revise and adjust the vision to obtain a visual balance or optimum spatial layout through 
the later phases of design in design environment. And it increased unnecessary time cost in design process.

\subsection{Motivation and Objective}

According to analysis above, this study proposed that if we can have customers' perspective of viewpoint in the process of sketch concept discussion to support the early phases of development of Show-Window sketch concept, not only is the time cost decreased in the later design process but more conceptual thinking is stimulated by variety of Multi-Perspective viewpoint. How to provide more and more viewpoint be referenced for designer in early phases of design that is our research focus? This study focus on Multi-Perspective viewpoint supported conceptual design sketch, so the design materials, design cost, design construction, and design illumination will not be discussed.

Therefore we present an interactive media of sketch-based that can get Multi-Perspective viewpoint in early phases of design to support designer. Further, designer not only can stimulate and generate more design thinking and design idea via continuing view but also can have synchronic interaction with object of the design environment. The interactive media can help designer changed the traditional way of Single-Perspective viewpoint into Multi-Perspective viewpoint in early phases of design, solved the drawback of Single-Perspective viewpoint. Thus it not only can decrease the blind spot of spatial vision by the traditional way of Single-Perspective viewpoint but also can increase more design thinking and decrease time cost in later phases of design.

\section{ANALYSIS}

\subsection{Sketch Media}

Sketching is not only an important part of evaluation in early phases of design but also a media that usually be used to represent concept. Sketching is a means of effective communication and creative concept development[7]. It is useful in setting that require a fast turn-around creation-feedback cycle, for example, during conceptual design[8]. And it is an external representation support for visualization and evaluation of the spatial arrangements of artifacts[9].

In Table 1, we can know the differences between traditional sketches and digital sketches[10]. This study makes integration good and bad into the interactive sketching media.

Table 1. The difference in sketch between traditional media and digital media

\begin{tabular}{|l|l|}
\hline Sketching on Paper & Digital Sketch \\
\hline $\begin{array}{l}\text { It does hard to modify as the design } \\
\text { evolves. }\end{array}$ & $\begin{array}{l}\text { It allows designers } \\
\text { to quickly record } \\
\text { design ideas in a } \\
\text { tangible form. }\end{array}$ \\
\hline $\begin{array}{l}\text { It may be annotated, but a designer } \\
\text { cannot easily search these }\end{array}$ & $\begin{array}{l}\text { It doesn't require } \\
\text { the designer to }\end{array}$ \\
\hline
\end{tabular}

\begin{tabular}{|l|l|}
\hline $\begin{array}{l}\text { annotations in the future to find out } \\
\text { why a particular design decision } \\
\text { was made. }\end{array}$ & $\begin{array}{l}\text { specify details that } \\
\text { may not yet be } \\
\text { known or important. }\end{array}$ \\
\hline $\begin{array}{l}\text { It is difficult to store, organize, } \\
\text { search, and reuse. }\end{array}$ & $\begin{array}{l}\text { It does easy to edit, } \\
\text { store, duplicate, } \\
\text { modify, and search. }\end{array}$ \\
\hline $\begin{array}{l}\text { It is difficult to store, organize, } \\
\text { search, and reuse. }\end{array}$ & \\
\hline $\begin{array}{l}\text { It is lack of interaction possible } \\
\text { between the paper-based design } \\
\text { and a user, which may be one of the } \\
\text { designers at this stage. }\end{array}$ & \\
\hline
\end{tabular}

In general, system supporting freehand sketching lack one or more of the properties that we believe a sketching system should have[11]: (1) It should be possible to draw arbitrary shapes with a single stroke, (i.e., without require the user to draw objects in pieces). (2) The system should do automatic feature point detection. The user should not have to specify vertex positions by hand. (3) The system should not have sketching modes for drawing different geometric object classes (i.e., modes for drawing circles, polylines, curves etc.). (4) The sketching systems should feel natural to the user.

Presenting the characteristics of traditional sketching media in digital ways, not only solving the difficulties in traditional media, but integrating the advantages in traditional sketching media to digital media, which makes the interactive sketching media easier to use and operate.

\subsection{Case Study}

In this section, a sketch-based case will be the body in the research, which will be divided into two parts. One part is the digital media to interact with the environment through interactive media. Another one is the digital media to transfer 2D stroke into 3D stroke.

(1) FreeDrawer: In Figure 3 right[12] is a sketching system for spline-based free-form surfaces on the Responsive Workbench. The paper proposes 3D tools for curve drawing and deformation techniques for curves and surfaces, adapted to the needs of designers. In this system, user can directly draws curves in the virtual environment, use a tracked stylus as input device. Another case in Figure 4 left[13] is a 3D painting system that allows one to draw lines, meshes and particle clouds using a one-hand wand in a virtual room with stereoscopic display.

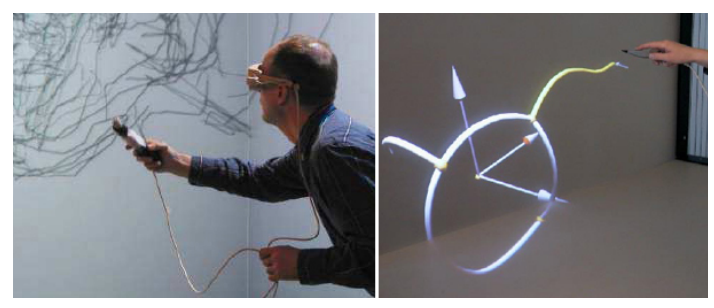

Figure 3. 3D painting system[13] and FreeDrawer[12] 
(2) Installation: In Figure 4[14] is a system for the creation of virtual form and their permanent installation into real space. It consisted of a viewing window (Figure 4) and stylus (Figure 4). The stylus and the viewing window were tracked in three dimensional position and orientation to calibrate virtual coordinate with real viewing position. Virtual objects created in the system responded as though they were physically in the space of the room.

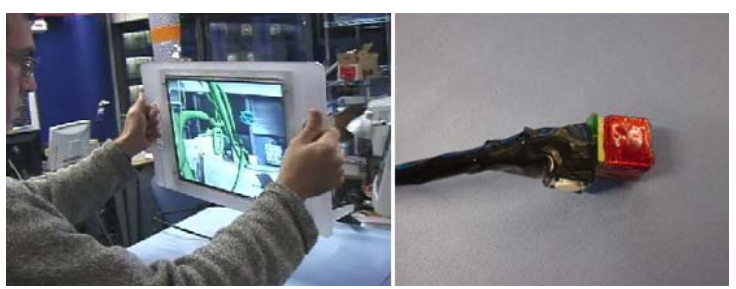

Figure 4. Media in design environment (left), and stylus (right)[14]

(3) Teddy: In Figure 5[15] presents a sketching interface for quickly and easily designing freeform models such as stuffed animals and other rotund objects. This paper uses 2D stoke to rapidly make 3D model and modified the 3D model object via 2D stroke. The system can paint, erase, extrude, cut, band, and smooth 3D model via 2D stroke.
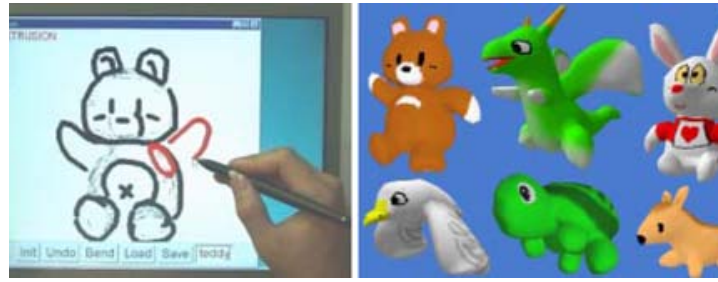

Figure 5. The system interface[15]

According to the investigating analysis above, we can realize via 3D stroke, the digital sketching media generates interaction with the environment, and then the user can immediately interact with the environment, and get instant perceptive feedback. In the analysis above, users can instantly modify their design, and preview the results of applying the design in the environment. Through the constantly examining the variation of conceptual design in the environment and modifying the conceptual design sketches, it can support users to stimulate their conceptual thinking and to generate the best sketches. This concept will be the foundation of researching interactive sketch media tools in this study.

\section{SYSTEM REQUIREMENT}

After analyzing the related works, we proposed the interactive concept diagram in Figure 6, which prefers that the user, through the interactive media, generates the interactive feedback with the design environment.

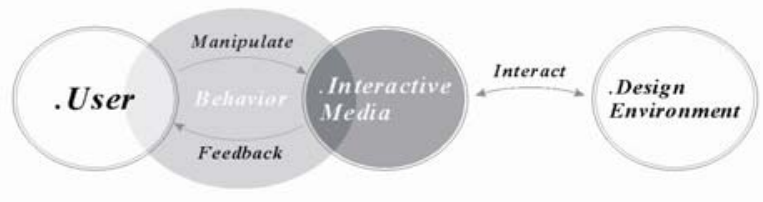

Figure 6. Interactive concept diagram

Through the interactive media to constantly examine the design environment, users get the conceptual information feedback. In the cycle users can repeatedly modify concepts and examine the interaction to obtain the optimum design concept.

After describing the system requirement above, we illustrated the interaction between the system and the design environment, and the functionality of each link in Figure 7, as our model of the implementation in this study.

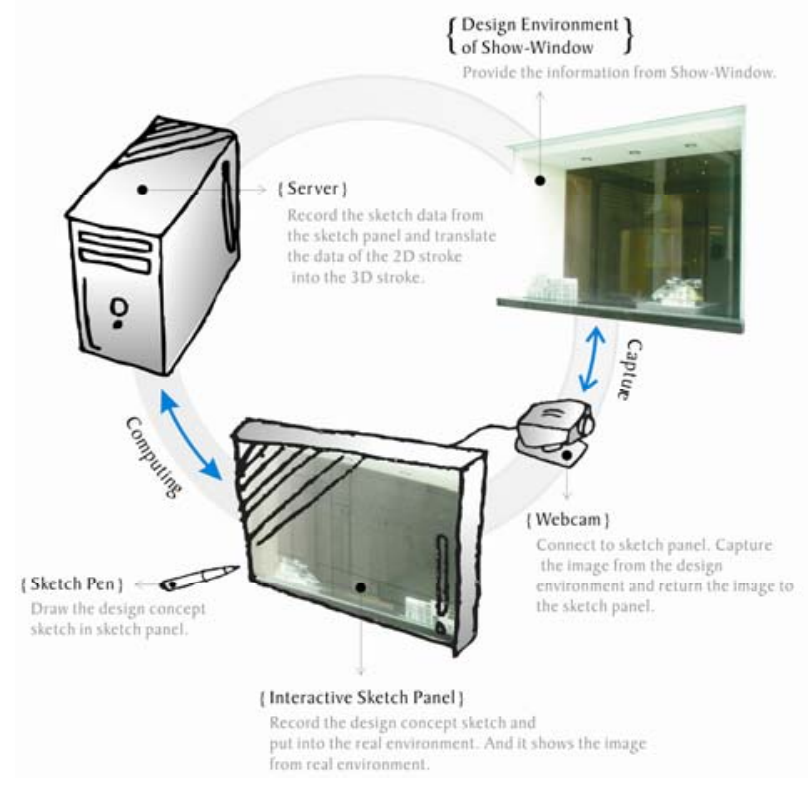

Figure 7. The system framework

After examining the interactive concept diagram, analyzing the case study and system framework, we proposed the design requirements based on the six sections below. We specified the interrelation and interaction between each, and constructed the system according to the six sections as the reliance of our implement.

(1) Pen-Based System: Freehand sketching is a familiar, efficient, and natural way of expression certain kinds of idea, particularly in the early phases of design[11]. Most of traditional conceptual sketch media use pen as their design concept transferring tool, which is the most direct and natural way to input. Through the pen-based input, design concepts are presented, so pen-based input is used in this study.

(2) 3D Stroke: Through computing, we transfer the 2D stroke sketch of the traditional media tool into 3D stroke. Once transferred into 3D stroke, it is convenient for users to examine the conceptual sketches and the viewpoint in 
space, and users can examine and interact with the design environment through different viewpoint.

(3) Transparent Layer: In the early phases of conceptual design sketches, sometimes we need to use annotation to record the ideas or announcements in design process for later discussion, and we usually need to repeatedly modify the conceptual sketches. In traditional sketch media, we usually add annotation or modify sketches on a transparent manuscript paper which can cover the original sketch, or directly do those on the original sketch. This study uses the transparent layer to record the design information that needs to be annotated or design concepts need to be modified.

(4) Direct Manipulate: This study will through intuitive manipulation to decrease the complex operating sequences, and make it as the reliance of our user interface design. The purpose is to decrease the variation on users' operating cognition and time cost to relearn the manipulation. Through this way, users can manipulate the interactive sketch media more intuitively and more conveniently to interact with the design environment.

(5) Immediate Feedback: The effects and variation generated by the interaction between the design environment and the user, through the instant reaction of the interactive sketch media, will be immediately returned to the user. Immediately obtaining the effect and variation in the design environment, they can immediately react to and modify them, and then give feedback to the interactive media to interact with the design environment. The optimization of the conceptual design sketches will be accomplished through continuously modifying, editing, and examining.

(6) Multi-Perspective: Transferring the traditional conceptual sketch drawing way via Single-Perspective viewpoint into Multi-Perspective viewpoint to examine the conceptual sketch. Multi-perspective viewpoint replaces the Single-Perspective viewpoint which supplements the unity in Single-Perspective viewpoint and provides the multiplicity in Multi-Perspective viewpoint. It not only solves the spatial visual blind spot problem, but also, via Multi-Perspective viewpoint, stimulates the design thinking and decreases the time cost in later phase of design.

\section{IMPLEMENTATION}

According to the analysis and the system framework (Figure 7) above, we divided the requirement to several parts into our system:

(1) Capture: The system can capture the image from design environment so we use the Webcam.

(2) Computing: The system need to have a server for computing so we need a Server.

(3) Draw Panel: The system need a platform to draw the design concept via sketch pen so we use a Touch Panel and Monitor Panel.

(4) Movable Perspective viewpoint: The system need have a movable perspective viewpoint for change the perspective viewpoint to get different perspective viewpoint so we use the device of Memsic 2152 to obtain data of move.

(5) Pen: The system need to have pen to draw the design concept on panel so we need a Sketch Pen.

According to requirement above, we design an interactive device (Figure 8).

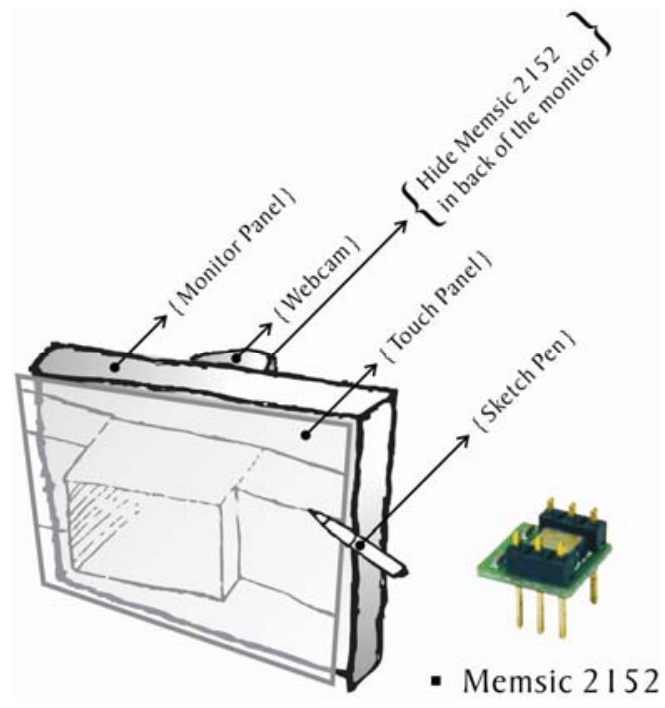

Figure 8. The device diagram

In Figure 8, Memsic 2125 is a device of the orientation that can gauge the data of the $\mathrm{X}$ axis and $\mathrm{Y}$ axis and return to server for compute the position. And we used the language of JAVA as a tool to implementation the system and connected these components above.

\section{SCENARIO}

We make a simple scenario to introduce the interactive media how to operate. Austin is a Show-Window designer, he takes this interactive media to sketch design concept while he come in Show-Window design environment. He takes the sketch pen to sketch design concept on the panel. He wants to change the viewpoint to examine the design sketch while he finishes the design concept, so he moves the panel and to start to change the viewpoint. Through the constantly examine the variation of conceptual design in the environment, he modifies the design concept while he thinks the design concept need to change. Finally he finishes the design concept sketch while he thinks the design concept is an optimal design concept.

\section{CONCLUSION}

This study developed an interactive media of Multi-Perspective viewpoint to support sketching. Breaking through the limit or traditional Single-Perspective viewpoint, not only makes the traditional design thinking model more flexible and selective, but also the sketch media, which provides designers the design thinking space 
and creativity, becomes more variable, changeable, and interactive.

\section{REFERENCES}

[1] Cuito A. Store Window Design. New York: TeNeues, 2005.

[2] Su Y-H. The Study of Influence between Different Elements in Window-Display and Image of Accessories- A Case of Aureate and Sliver Accessories. Department in Visual Communication Design. Douliou: National Yunlin University of Science and Technology, 2005. pp. 6.

[3] Huang S-H, Wu R-F. Exhibition Design. Taipei: San-Ming, 1992.

[4] Junichi N. Color Theory of Merchandise. Tokyo, 1985.

[5] Lin B-L. Color Design in Show-Window Disply. Tainan: Shin-Hung, 1993.

[6] Chiaki K. Appearance to reveal design. Taipei: Art Technology Co.,Ltd, 2001.

[7] Nam TJ. Sketch-Based Rapid Prototyping Platform for Hardware-Software Integrated Interactive Products. International Conference for Human-Computer Interaction. Portland, Oregon USA: ACM, 2005. pp. 1689-92.

[8] Do EY-L. VR Sketchpad. CAAD Future. Netherlands: KLUWER ACADEMIC, 2001. pp. 161-72.

[9] Do EY-L. Design Sketches and Sketch Design Tools. Knowledge-Based Systems 2005;383-405:383-404.

[10] Landay JA, Myers BA. Interactive Sketching for the Early Stages of User Interface Design. Conference on Human Factors in Computing Systems. Denver, Colorado, USA: ACM, 1995.

[11] Sezgin TM, Davis TSR. Sketch Based Interfaces: Early Processing for Sketch Understanding. Perceptive User Interfaces. Orlando, FL, USA, 2001. pp. 1-8.

[12] Wesche G, Seidel H-P. FreeDrawer - A Free-Form Sketching System on the Resposive Workbench. Virtual Reality Software \& Technology. Banff, Alberta, Canada: ACM, 2001. pp. 167-74.

[13] Makela W, Takala MRT. Possibilities and Limitations of Immersive Free-Hand Expression: a Case Study with Professional Artists. ACM Multimedia. New York, USA: ACM, 2004. pp. 504-7.

[14] Greemwold S. Spatial Computing. Graduate School of Science in Media Arts and Sciences. Massachusetts: Massachusetts Institute of Technology, 2003. pp. 47-61.

[15] Igarashi T, Matsuoka S, Tanaka H. Teddy: A Sketching Interface for 3D Freeform Design. SIGGRAPH. Los Angeles, California, USA: ACM, 1999. 